\title{
Rhizobacteria as bioenhancer and biofertilizer for growth and yield of banana (Musa spp. cv. 'Berangan')
}

\begin{abstract}
Banana, an important fruit crop, requires high amounts of fertilizers for commercial cultivation, which is costly and can be hazardous to the environment when used excessively. Inoculations of plant growth promoting rhizobacteria (PGPR) have been shown to produce beneficial effects through growth stimulation in legumes and cereals, and an attempt has been made to use rhizobacteria in bananas. Three experiments were conducted to observe the effects of plant growth promoting rhizobacteria (PGPR) inoculation on root stimulation and colonization, nutrient absorption, growth and yield of bananas (Musa spp. cv. 'Berangan', AA type). The results showed that inoculation together with $33 \% \mathrm{~N}$ improved the bioenhancing activity by increasing root and shoot growth, and photosynthetic rate. The PGPR inoculation with $33 \% \mathrm{~N}$-fertilizer also increased the Ca uptake capacity resulting in higher $\mathrm{Ca}$ concentration in root, corm and pulp but increased the $\mathrm{Mg}$ concentration in the root only. In addition, the total accumulation of nutrients was heavily influenced by PGPR inoculation due to enhanced root proliferation. The PGPR inoculation greatly increased the bunch yield (35-51\%). The strains Sp7 and UPMB10 were evaluated for their N2 fixing capacities in association with banana roots by $15 \mathrm{~N}$ isotopic dilution technique and acetylene reduction assay (ARA). The results conclusively showed that roots of PGPR-inoculated plants fixed N2 and produced higher ARA values and inoculated plants together with the least $\mathrm{N}$-fertilizer supply showed the highest amount of nitrogen derived from atmosphere (Ndfa). The findings from the above studies demonstrated that PGPR strains (Sp7 and UPMB 10) inoculation with minimal $\mathrm{N}$-fertilizer supply are effective as a bioenhancer for increasing plant growth, nutrient uptake and yield of bananas.
\end{abstract}

Keyword: Banana, Rhizobacteria, Bioenhancer, Growth, Yield 